\title{
Renal involvement in overlap syndrome
}

\author{
Sineta Cristina Firulescu', Paulina Lucia Ciurea'2, Cristina Dorina Parvanescu', \\ Beatrice Andreea Chisalau', Andreea Lili Barbulescu' ${ }^{3}$, Simona Banicioiu-Covei', \\ Stefan Cristian Dinescu'2, Florentin Ananu Vreju \\ ${ }^{1}$ University of Medicine and Pharmacy, Craiova, Romania \\ ${ }^{2}$ Department of Rheumatology, University of Medicine and Pharmacy, Craiova, Romania \\ ${ }^{3}$ Department of Pharmacology, University of Medicine and Pharmacy, Craiova, Romania
}

\begin{abstract}
Overlap syndrome (OS) is a clinical condition that fulfils the criteria of at least two conjunctive tissue diseases (CTD) occurring in the same patient at the same time or at different moments and its complex clinical picture often makes the diagnosis difficult.

Aim. The aim of the study was to evaluate the frequency of renal involvement in a group of patients with overlap syndrome, compared to single CTD.

Methods. The observational retrospective study included 122 patients diagnosed with CTD and OS, admitted into Rheumatology Clinic of the Emergency County Clinical Hospital Craiova, between 2011 and 2018, with similar mean age for the two groups (51.41 \pm 10.88 yo for OS group and $50.78 \pm 12.65$ yo for the other).

Results. The proteinuria was significantly lower in the OS group $8 \%$ in comparison to the ones without OS (20\%) $(p=0.044)$. The presence of SLE disease and its immunologic changes seems to impact negatively the kidneys, no matter the disease that is associated. Thus, we noticed the presence of renal disease in a percentage of $50 \%$ for the patients that associated SLE to SSc, 25\% for the ones with SLE and MTCD and 27\% for SLE-RA. Conclusions. Although the frequency of renal disease is lower in case of OS, it is important to be recognized, appropriately monitored and integrated in therapeutic decision, especially that some associations are more prone to develop kidney involvement.
\end{abstract}

Keywords: connective tissue disease, proteinuria, renal involvement, overlap syndrome

\section{INTRODUCTION}

Overlap syndrome (OS) is a clinical condition that fulfils the criteria of at least two conjunctive tissue diseases (CTD) occurring at the same time or at different moments for the same patient [1]. OS may associate any of the following: systemic scleroderma (SSc), dermatomyositis or polymyositis (DM/PM), Sjögren's syndrome (SS), rheumatoid arthritis (RA), and systemic lupus erythematosus (SLE) in any manner, with certain possibilities being more common. The complex clinical picture often makes the diagnosis difficult. The pathogenesis of each of these conditions has a major impact on the overlap syndrome's clinical development, diagnosis and treatment [2]. The symptoms depend on the organ involvement and disease duration. Clinical manifestations in overlap connective tissue diseases such as skin, digestive, hematological, pulmonary or renal disease, associated or not to several immunological changes, may be more or less expressed.

In CTD, renal involvement may imply major consequences and unfavorable prognosis, which requires aggressive and immediate treatment. Renal impairment may be related to both disease evolution and therapeutic approach. It may be asymptomatic or can be expressed as life-threatening renal failure.

\begin{abstract}
AIM
The aim of the study was to evaluate the frequency of renal involvement in a group of patients with overlap syndrome, compared to single CTD, and its possible inter-relation with immunologic changes.
\end{abstract}




\section{METHODS}

We performed an observational retrospective study that included 122 patients diagnosed with CTD and OS, admitted into Rheumatology Clinic of the Emergency County Clinical Hospital Craiova, between 2011 and 2018. The data included demographic information, disease duration, disease signs and symptoms, joint involvement, systemic manifestations, laboratory and imaging findings. All patients have fulfilled the diagnostic criteria for SLE, RA, SSc, SS and the Bohan and Peter's criteria for $\mathrm{DM} / \mathrm{PM}$

The study was performed in accordance with the Declaration of Helsinki and approved by the Ethics Committee of the University of Medicine and Pharmacy of Craiova. All patients provided their written informed consent, after receiving a standard form which mentioned that the results would be used for research purposes.

\section{RESULTS}

The mean age was similar for the two groups, approximately 51 years $(51.41 \pm 10.88$ yo for OS group and $50.78 \pm 12.65$ yo for the other). The female gender is exclusively present $(100 \%)$ in our OS patients and has a percentage of $85 \%$ in the group without OS. The disease duration under 10 years is similar for both groups, OS and CTD patients (9.14\% vs. $10.17 \%$ ).

Demographic characteristics of the study group are presented in table 1 .

TABLE 1. Demographic characteristics of the study group

\begin{tabular}{|l|c|c|}
\hline & OS & Without OS \\
\hline Age & & \\
Mean (SD) & $51.41(10.88)$ & $50.78(12.65)$ \\
Min & 28 & 27 \\
Max & 80 & 76 \\
$<55$ years (N,\%) & $36,56 \%$ & $36,61 \%$ \\
$>55$ years (N,\%) & $28,44 \%$ & $23,39 \%$ \\
\hline Sex & & \\
Women (N,\%) & $64,100 \%$ & $50,85 \%$ \\
Men (N,\%) & $0,0 \%$ & $9,15 \%$ \\
\hline Disease duration & & \\
(years; N,\%) & & \\
$<2$ & $9,14 \%$ & $22,37 \%$ \\
$2-5$ & $24,38 \%$ & $15,25 \%$ \\
$5-10$ & $22,34 \%$ & $12,20 \%$ \\
$>10$ & $9,14 \%$ & $10,17 \%$ \\
\hline
\end{tabular}

Of the 59 patients with single CTD, 20 patients were with SLE, 11 were diagnosed with DM/PM, 21 with SSc and 7 patients with SS.
Out of the 63 patients with OS, 37 patients associated RA with another CTD (58.73\%), 19 patients SLE associated with another CTD (36.16\%), 1 patients SSc-DM and 1SSc-SS; the association of 3 or 4 CTD was reckoned for 5 patients.

Renal involvement was evaluated by the presence of proteinuria and the value for serum urea and creatinine. The percentage calculated for proteinuria in our two study groups are described in table 2 .

TABLE 2. Frequency of renal involvement (proteinuria)

\begin{tabular}{|c|c|c|c|c|}
\hline & $\begin{array}{c}\text { With renal } \\
\text { involvement } \\
\text { (proteinuria) } \\
\text { N (\%) }\end{array}$ & $\begin{array}{c}\text { Without renal } \\
\text { involvement } \\
\text { (proteinuria) } \\
\mathrm{N}(\%)\end{array}$ & Total & $\begin{array}{l}\text { Chi- } \\
\text { Square } \\
\text { Test }\end{array}$ \\
\hline OS & $5(8 \%)$ & 59 (92\%) & 64 & \multirow{3}{*}{$p=0.044$} \\
\hline $\begin{array}{l}\text { Without } \\
\text { OS }\end{array}$ & 12 (20\%) & 47 (80\%) & 59 & \\
\hline Total & 17 & 106 & 123 & \\
\hline
\end{tabular}

As can be observed, the proteinuria is present for $8 \%$ of the patients with OS and for $20 \%$ of the ones without OS, $\mathrm{p}=0.044$ (table 2, figure 1).

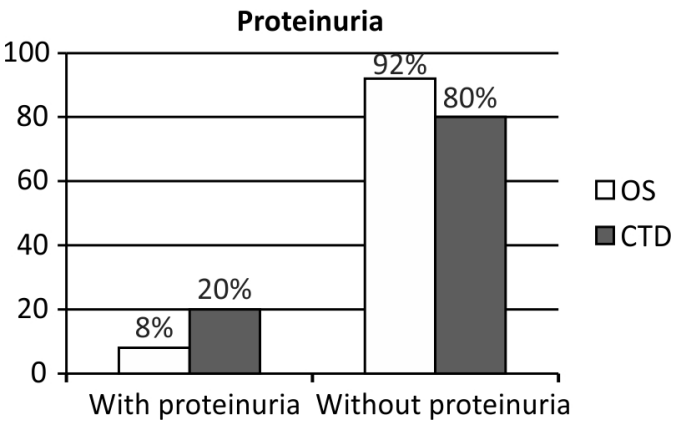

FIGURE 1. Frequency of renal involvement (proteinuria)

Analyzing the type of OS and the relationship with renal involvement, depending on the CTD association, we found proteinuria in $27.27 \%$ of the patients with SLE and RA, compared to a higher percentage of $55 \%$ calculated for patients only with SLE (figure 2).

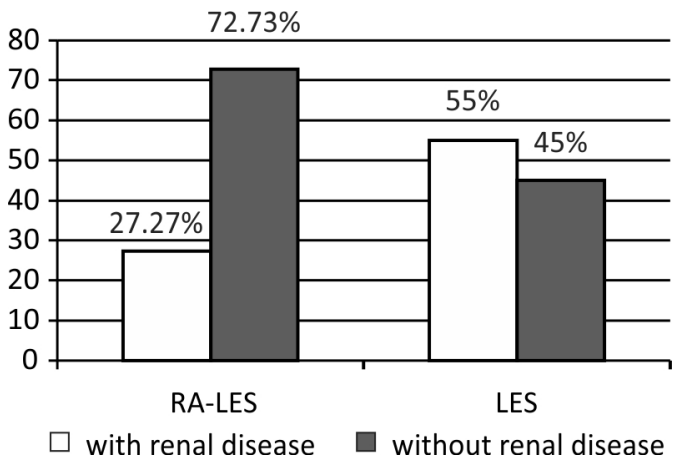

FIGURE 2. The presence of renal involvement in $R A-S L E / S L E$ patients 
The presence of rheumatoid factor (RF) seems to decrease in some measure the renal involvement, both in OS and in different single CTD. Thus, FR presence within SLE-RA overlap syndrome, imprinted a decrease of kidney disease to $9 \%$ (figure 3 ).

TABLE 3. RF association with the presence of proteinuria in patients with OS

\begin{tabular}{|l|c|c|c|}
\hline & $\begin{array}{c}\text { Renal } \\
\text { involvement } \\
\text { (proteinuria) } \\
\mathbf{N}(\%)\end{array}$ & $\begin{array}{c}\text { Renal } \\
\text { involvement - } \\
\text { RF positive, N }\end{array}$ & $\begin{array}{c}\text { Total } \\
\text { patients }\end{array}$ \\
\hline SLE-RA & $3(27 \%)$ & $1(9 \%)$ & 11 \\
\hline SLE-BMTC & $1(25 \%)$ & $1(25 \%)$ & 4 \\
\hline $\begin{array}{l}\text { SLE-SSc } \\
\text { (limited form) }\end{array}$ & $1(50 \%)$ & 0 & 2 \\
\hline Total (OS) & $\mathbf{5 ( 7 . 8 \% )}$ & $\mathbf{2 ( 3 . 1 2 \% )}$ & $\mathbf{6 4}$ \\
\hline
\end{tabular}

\begin{tabular}{|l|c|c|c|}
\hline SLE & $11(55 \%)$ & 0 & 20 \\
\hline DM/PM & $1(9 \%)$ & $1(9 \%)$ & 11 \\
\hline Total (CTD) & $\mathbf{1 2 ( 2 0 . 3 3 \% )}$ & $\mathbf{4 ( 6 . 7 7 \% )}$ & $\mathbf{5 9}$ \\
\hline
\end{tabular}

The presence of SLE disease and its immunologic changes seems to impact negatively the kidneys, no matter the disease that is associated. Thus, we noticed the presence of renal disease in a percentage of $50 \%$ for the patients that associated SLE to SSc, $25 \%$ for the ones with SLE and MTCD and 27\% for SLE-RA.

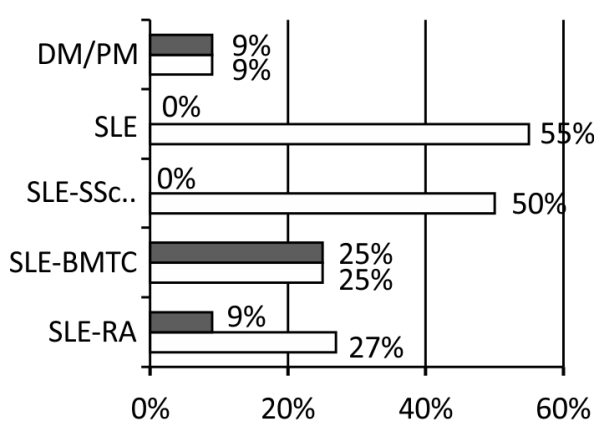

$\square$ Renal involvement and RF+

$\square$ Renal involvement

FIGURE 3. Distribution of renal involvement and its association with RF

\section{DISCUSSIONS}

CTD, characterized by multisystemic involvement and immune dysregulation, can frequently associate renal involvement, with major consequences and unfavorable prognosis that usually imposes an aggressive treatment. The literature describes its presence in SLE in percentages ranging from 20 to $55 \%$, in SLE, RA, DM/PM or MCTD, being higher in SSc (60-80\%), and lower for SS patients (5-71\%) [3-6]. Several scientific reports concluded that, compared to pure disease, in OS, the renal impairment has a lower frequency, conclusions consistent with our research. Thus, our results describe a higher percentage of renal involvement in patients with only SLE, compared to OS that includes SLE, either with RA, SSc or MCTD. In a similar manner, Manoussakis et al. published an analysis of a 26 patients group with SLE-SS association, with only $11.5 \%$ that had kidney disease compared to $55 \%$ of the patients with SLE only [4]. Also, Hai Feng Pan et al. reported a percentage of $48 \%$ for SLE-SS patients and $66.7 \%$ for SLE ones [7].

In the same manner, the RA-SLE association, entity called rhupus, displays a lower renal disease compared to SLE patients, observation made by Jing $\mathrm{Li}$ et al., analyzing 56 patients with rhupus versus 160 SLE subjects [8].

SSc association with any of PM/DM, SLE or RA, determines a delay for the onset of renal scleroderma crisis; in the study of Foocharoen $\mathrm{C}$ et al., that included 68 patients with the SSc associated with different diseases (PM, SLE and RA), the prevalence of renal scleroderma crisis was similar in the SSc-PM lot, compared to pure SSc group (2.1\%), the other associations showing no renal impairment [9]. Otherwise, patients with only PM or DM seems to be protected against renal impairment, in comparison to OS that associates DM/PM and any MTCD, such as SSc, SLE, RA or SS, $(3.7 \%$ and $1.9 \%$ respectively vs. $13.4 \%)[10]$.

\section{CONCLUSIONS}

Renal involvement is common in patients with different CTD and OS and its early diagnosis is essential in order to prevent further complications which can alter the prognosis and have an unfavorable input on patient's quality of life. Although the frequency of renal disease is lower in case of OS, it is important to be recognized, appropriately monitored and integrated in therapeutic decision, especially that some associations are more prone to develop kidney involvement.

Conflict of interest: none declared Financial support: none declared 


\section{REFERENCES}

1. laccarino L, Gatto M, Bettio S, Caso F et al. Overlap connective tissue disease syndromes. Autoimmun Rev. 2013;12(3):363-73.

2. Balbir-Gurman $A$, Braun-Moscovici Y. Scleroderma overlap syndrome. Isr Med Assoc J. 2011;13(1):14-20.

3. Mittal T, Rathi M. Rheumatological diseases and kidneys: A nephrologist's perspective. Int J Rheum Dis. 2014;17(8):834-44.

4. Jain A, Srinivas BH, Emmanuel D, Jain VK, Parameshwaran S, Negi VS. Renal involvement in primary Sjögren's syndrome: A prospective cohort study. Rheumatol Int. 2018;38(12):2251-2262.

5. Kitridou $\mathrm{RC}$ et al. Renal involvement in mixed connective tissue disease: A longitudinal clinicopathologic study. Semin Arthritis Rheum. 1986;16(2):135-45.

6. Couderc M et al. Prevalence of Renal Impairment in Patients With Rheumatoid Arthritis: Results From a Cross-Sectional Multicenter Study. Arthritis Care Res (Hoboken). 2016;68(5):638-44.
7. Pan HF et al. Clinical and laboratory profiles of systemic lupus erythematosus associated with Sjögren's syndrome in China: a study of 542 patients. Clin Rheumatol. 2008;27(3):339-43.

8. Li J et al. Clinical analysis of 56 patients with rhupus syndrome: Manifestations and comparisons with systemic lupus erythematosus - a retrospective case-control study. Medicine (Baltimore). 2014;93(10):e49.

9. Foocharoen C, Netwijitpan S, Mahakkanukrauh A, Suwannaroj S, Nanagara R. Clinical characteristics of scleroderma overlap syndromes: Comparisons with pure scleroderma. Int J Rheum Dis. 2016;19(9):913-23.

10. Nuño-Nuño $L$ et al. Overlap myositis, a distinct entity beyond primary inflammatory myositis: A retrospective analysis of a large cohort from the REMICAM registry. Int $J$ Rheum Dis. 2019;22(8):1393-1401. 